\title{
Optimum design of fatigue-resistant composite laminates using hybrid algorithm
}

\author{
H. Arda Deveci ${ }^{a, b}$, H. Seçil Artem ${ }^{a, *}$ \\ ${ }^{a}$ Department of Mechanical Engineering, İzmir Institute of Technology, Gülbahçe Kampüsü, 35430 Urla, İzmir, Turkey \\ ${ }^{\mathrm{b}}$ Department of Mechanical Engineering, Erzincan University, Yalnızbağ Yerleşkesi, Erzincan, Turkey
}

\section{A R T I C L E I N F O}

\section{Article history:}

Received 14 October 2016

Revised 12 January 2017

Accepted 28 January 2017

Available online 8 February 2017

\section{Keywords:}

Laminated composites

Fatigue

Life prediction

Optimization

Hybrid algorithm

\begin{abstract}
A B S T R A C T
In this study, a fatigue life prediction model termed as Failure Tensor Polynomial in Fatigue (FTPF) is applied to the optimum stacking sequence design of laminated composites under various in-plane cyclic loadings to obtain maximum fatigue life. The validity of the model is investigated with an experimental correlation using the data available in the literature. The correlation study indicates the reliability of FTPF, and its applicability to different composite materials and multidirectional laminates. In the optimization, a hybrid algorithm combining genetic algorithm and generalized pattern search algorithm is used. It is found by test problems that the hybrid algorithm shows superior performance in finding global optima compared to the so far best results in the literature. After the verifications, a number of problems including different design cases are solved, and the optimum designs constituted of discrete fiber angles which give the maximum possible fatigue lives are proposed to discuss. A comparison study is also performed with selected design cases to demonstrate potential advantages of using non-conventional fiber angles in design.
\end{abstract}

(C) 2017 Elsevier Ltd. All rights reserved.

\section{Introduction}

Fiber reinforced composites have increasingly been used instead of conventional metallic materials in industries such as aviation, aerospace, marine and automotive due to their advantageous properties like high strength, high stiffness, and low weight. Fatigue is one of the critical failure mechanisms in composite structures subjected to cyclic loading in which crack formation and propagation finally lead to fatigue failure which may cause the loss of structural integrity. Therefore, it is important to consider fatigue in investigation and design processes of the composite structures that must bear significant cyclic fatigue loads during operation, such as airplanes, wind turbine rotor blades, boats and bridges [1].

Composite structures offer great design flexibility since they enable material tailoring in different ways to meet specific design requirements. Fatigue strength, thus structural performance of laminated composites can significantly be increased through the optimum selection of parameters such as fiber and matrix materials, fiber orientations or layer thicknesses. Although there are many studies related to optimization of laminated composites subjected to static loadings in the literature (i.e., weight minimization,

\footnotetext{
* Corresponding author.

E-mail address: secilartem@iyte.edu.tr (H.S. Artem).
}

buckling strength maximization, etc.), there are very few published studies on the optimum design of laminated composites under fatigue loading [2-5]. As a pioneering study in this field, Adali [2] optimized a symmetric angle-ply laminate under in-plane tensile fatigue loads for maximum failure load by employing a fatigue failure criterion, and determined optimum fiber orientations, thickness ratio and fiber content for constant cyclic lives. Walker [3] presented a procedure to minimize the thickness of laminated composite plates subjected to cyclic bending loads for specific fatigue lives under a cumulative damage constraint. In the previous studies on fatigue design optimization, the researchers used fatigue models that were valid only for limited laminate configurations and specific loading conditions. Essentially, more general stacking sequences and loading conditions need to be considered in design optimization for typical applications. In this regard, the study of Ertas and Sonmez [4] showed that the optimum designs of laminated composite plates under in-plane cyclic loading for maximum fatigue life can theoretically be obtained for more general stacking sequences. More recently, Muc and Wierzgoń [5] proposed a design methodology to find the optimum stacking sequences having three different fiber orientations for maximum buckling load of composite plates by introducing a new type of discrete design variables.

Fatigue life prediction of composite materials enables to be able to design fatigue-resistant composite structures. A large number of 
fatigue theories and methodologies for the fatigue life prediction of composite materials and structures have been developed so far in the literature, based on empirical, phenomenological modelling; specific damage metrics such as the residual strength and/or stiffness of the examined material; probabilistic; artificial neural network based; and micromechanics. Moreover, the investigation of fatigue behavior of composite plates under multiaxial loadings is more important for the applications subjected to real complex loading conditions [6]. Among these many fatigue life prediction models for composites, most of them are not suitable for optimum design of laminated composites due to their limited applicability. In this respect, it is reported that empirical models which estimate the fatigue life due to constant amplitude loading based on experimental data without making any assumption regarding the micro mechanisms leading to fatigue failure have practical application potential in fatigue design of composite structures [1]. For example, Hashin and Rotem [7] presented such an experimental fatigue life prediction model based on the different failure modes which exist for unidirectional materials, the fiber and matrix failure modes. The authors reported that an interlaminar failure mode is encountered when multidirectional composite laminates are considered [8]. Fawaz and Ellyin [9] proposed a multiaxial fatigue life prediction model based on Tsai-Hill static strength failure criterion, requiring only an experimental S-N curve of a reference offaxis specimen. They indicated that the model accurately predicts fatigue failure of different unidirectional and bidirectional fiber reinforced composite materials subjected to uniaxial and multiaxial stresses and different cyclic stress ratios. Philippidis and Vassilopoulos [10] introduced a model which is an extension of the quadratic version of the Failure Tensor Polynomial for the prediction of fatigue life under complex stress states. It is reported that the model yields reliable predictions for both unidirectional and multidirectional laminated composites when compared to experimental data, and can be used in design of composite structures subjected to multiaxial fatigue loadings. Kawai [11] developed a phenomenological fatigue damage mechanics based model that could take into account the off-axis angle and stress ratio effect under any constant amplitude loading with non-negative mean stresses in order to predict the off-axis fatigue behavior of unidirectional composites. It is shown that the model is capable of adequately predicting the off-axis behavior over a range of nonnegative mean stresses.

In the present study, the fatigue life prediction model of Philippidis and Vassilopoulos [10] is preferred for both the prediction capability on multidirectional composite laminates and the ease of applicability. A methodology is proposed to find optimum stacking sequence designs of laminated composites with maximum fatigue life under different in-plane cyclic loadings. The model is used to constitute the objective function, and a hybrid generalized pattern search embedded genetic algorithm is used in the optimization. Before the optimization, a comprehensive experimental correlation study is performed for different unidirectional and multidirectional composite materials to validate the model. Then, the effective performance of the proposed hybrid algorithm is shown using a test problem from the literature. After these investigations, a number of problems including different design cases are solved and the optimum results are presented to discuss. Also, static failure indexes of the designs according to Hashin-Rotem failure criterion [12] are determined and given with the results.

Among the optimization studies under fatigue loading in the literature [2-5], there aren't any studies which verify a fatigue life prediction model for different composite materials and multidirectional laminates, and use this model to find optimum fatigueresistant stacking sequence designs by a hybrid algorithm. Furthermore, the literature is deficient in studies considering hybrid algorithms for the optimization of laminated composites. In this regard, this study fulfils the gap in the literature and reveals the potential for improvement of fatigue life of laminated composites.

\section{Fatigue life prediction model}

Design optimization of composite laminates for maximum fatigue strength requires a reliable fatigue assessment model that accounts for the factors affecting fatigue life. The fatigue strength of a given fiber-reinforced composite laminate mainly depends on the type of constituent layers and the fiber orientation angle $(\theta)$ with respect to the loading directions, and magnitude of the cyclic stress as cyclic stress ratio. Besides these mentioned requirements, the option of the method to use laminate properties instead of lamina properties to predict laminate behavior enhances the applicability of the criterion for unidirectional (UD) and multidirectional lay-ups made up of any type of composite, e.g., UD, woven or stitched layers. Also, in order to reduce the experimental effort, the method should require few empirical relations as reference for the predictions. In these respects, failure tensor polynomial based method of Philippidis and Vassilopoulos [10,13] seems to be a promising model to use in a design optimization study when considering its applicability to various in-plane loading states and arbitrary fiber orientations requiring little experimental effort.

\subsection{Failure tensor polynomial in fatigue (FTPF) criterion}

A modification of the quadratic version of the failure tensor polynomial for the prediction of fatigue strength under complex stress states was introduced by Philippidis and Vassilopoulos [10] and termed as Failure Tensor Polynomial in Fatigue (FTPF) criterion. The FTPF criterion is based on Tsai-Hahn tensor polynomial [14] and adapted for fatigue.

For a fiber-reinforced composite plate subjected to in-plane loading (Fig. 1), Tsai-Hahn tensor polynomial criterion is expressed in the material coordinates by

$F_{11} \sigma_{1}^{2}+F_{22} \sigma_{2}^{2}+2 F_{12} \sigma_{1} \sigma_{2}+F_{1} \sigma_{1}+F_{2} \sigma_{2}+F_{66} \sigma_{6}^{2}-1 \leqslant 0$

Here, the components of the failure tensors can be given by

$$
\begin{aligned}
& F_{11}=\frac{1}{X X^{\prime}}, \quad F_{22}=\frac{1}{Y Y^{\prime}}, \quad F_{66}=\frac{1}{S^{2}}, \quad F_{1}=\frac{1}{X}-\frac{1}{X^{\prime}}, \quad F_{2}=\frac{1}{Y}-\frac{1}{Y^{\prime}}, \\
& F_{12}=-\frac{1}{2} \sqrt{F_{11} F_{22}}
\end{aligned}
$$

where $X$ and $Y$ represent the failure strengths of the material along the longitudinal and the transverse directions, respectively, and $S$ represents shear failure strength. The prime (') is used for compressive strengths.

For cyclic in-plane fatigue loading, the components of failure tensors are functions of the number of cycles $N$, stress ratio, $R=\sigma_{\min } / \sigma_{\max }$, and the frequency $v$, of the loading as

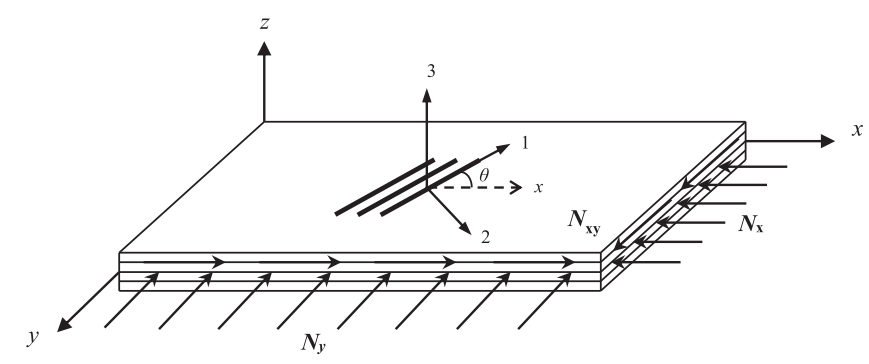

Fig. 1. Representative plate geometry showing in-plane loading and principal coordinates. 
$F_{i j}=F_{i j}(N, R, v), \quad F_{i}=F_{i}(N, R, v), \quad i, j=1,2,6$

and the expressions in Eq. (2) are still valid for the calculation of tensor components but the failure stresses $X, X^{\prime}, Y, Y^{\prime}$, and $S$ are replaced by the $\mathrm{S}-\mathrm{N}$ curves of the material along the same directions and under the same conditions. Thus, the failure stresses $X, X^{\prime}, Y, Y^{\prime}$, $S$ can be expressed as functions of number of cycles, stress ratio and frequency. If the S-N curves of the material are assumed in the general semi-logarithmic form

$S=A+B \log N$

then, the expressions of the fatigue failure stresses can be written as

$$
\begin{aligned}
& X(N, R, v)=A_{X}+B_{X} \log N \\
& X^{\prime}(N, R, v)=A_{X^{\prime}}+B_{X^{\prime}} \log N \\
& Y(N, R, v)=A_{Y}+B_{Y} \log N \\
& Y^{\prime}(N, R, v)=A_{Y^{\prime}}+B_{Y^{\prime}} \log N \\
& S(N, R, v)=A_{S}+B_{S} \log N
\end{aligned}
$$

For the composite materials in which static tensile strengths are equal or close to static compressive strengths, it is assumed that $X^{\prime}=X$ and $Y^{\prime}=Y$, and only $X(N, R, v), Y(N, R, v)$ and $S(N, R, v)$ from the above fatigue failure stresses are sufficient for the FTPF criterion to yield satisfactory predictions. When only these three S-N curves are used, the fatigue failure tensor components can be given by

$$
\begin{aligned}
& F_{11}=\frac{1}{X^{2}(N, R, v)}, \quad F_{22}=\frac{1}{Y^{2}(N, R, v)}, \quad F_{66}=\frac{1}{S^{2}(N, R, v)}, \\
& F_{1}=F_{2}=0
\end{aligned}
$$

and by substituting these tensors into Eq. (1), the criterion finally takes the form

$\frac{\sigma_{1}^{2}}{X^{2}(N)}+\frac{\sigma_{2}^{2}}{Y^{2}(N)}-\frac{\sigma_{1} \sigma_{2}}{X(N) Y(N)}+\frac{\sigma_{6}^{2}}{S^{2}(N)}-1 \leqslant 0$

where the fatigue failure stresses are shown only as functions of the number of cycles $N$. The criterion can be used in the form of Eq. (7) for any stress ratio $R$, and frequency $v$, provided the basic $\mathrm{S}$-N curves are also known for the same $R$ and $v$ values.

\subsection{Experimental correlation of the criterion}

Philippidis and Vassilopoulos [10] demonstrated the applicability of the FTPF criterion on various fiber reinforced composites under uniaxial and biaxial loading conditions by comparing the predictions of their model with the experimental data available in the literature. It is reported that the FTPF criterion can be used for realistic design cases of fiber reinforced composites with unidirectional and multidirectional lay-ups made up of any type of composite layers (e.g., UD, woven or stitched) under multiaxial stress states. In the study, the agreement between the model's predictions and experimental data is found satisfactory or at least with the same accuracy as two other fatigue life prediction models $[7,9]$ for off-axis angles of the laminates. However, directly onaxis predictions of the FTPF criterion for multidirectional laminates are not demonstrated in details.

In this study, the FTPF criterion has been applied to find optimum multidirectional laminates consisting of different fiber alignments according to different designs cases. Hence, it is important to understand whether the criterion can perform accurate fatigue life estimations of multidirectional laminates. In this regard, prediction capability of the criterion on different composite materials in various multidirectional stacking sequences is investigated by a procedure taking multiaxial stress states into consideration. The procedure consists of two parts. The first part is related to the determination of the fatigue load range to be applied for the prediction. For this purpose, first, logarithmic fatigue lives $((\log N))$ corresponding to minimum and maximum stress amplitudes $\left(\sigma_{\mathrm{a}}\right)$ are determined from the experimental data for the related laminate. Then, minimum and maximum cyclic load values corresponding to these fatigue lives are found by solving the FTPF criterion equation (Eq. (7)) for the outmost layer of the laminate. The outmost layer is considered in calculations since measurements in the experiments are taken from the outmost layer. Note that the principal stresses appear in Eq. (7) are calculated using the classical lamination theory (CLT). In the second part of the procedure, fatigue life of the laminate is simulated between the stress amplitude range using a formula including $\log N$ parameter. This formula is obtained for each considered laminate by substituting Eq. (5) into Eq. (7) and shown in closed form below

$f\left(\sigma_{1}, \sigma_{2}, \sigma_{6}, \log N\right)-1 \leqslant 0$

For the related laminate, first, based on the outermost layer, stress amplitude range are calculated from the minimum and maximum loads determined in the first part using the CLT. Afterwards, $\log N$ values corresponding to these principal stresses are obtained by solving Eq. (8). Thus, fatigue life for a given laminate is estimated.

Four different fiber reinforced composite materials in various multidirectional stacking sequences selected from the literature [15-18] are examined for the life prediction. Moreover, regression analysis is performed for each laminate to demonstrate how good the prediction curve fits the data. Therefore, the coefficient of determination $\left(R^{2}\right)$ is specified for each prediction. The coefficient of determination is expressed with the following equation

$R^{2}=\frac{S S_{R}}{S S_{T}}$

where $S S_{R}=\sum_{i=1}^{n}\left(\hat{y}_{i}-\bar{y}\right)^{2}$ which is called the regression sum of squares, $S S_{T}=\sum_{i=1}^{n}\left(y_{i}-\bar{y}\right)^{2}$ which is called the total sum of squares. Here, $y_{i}$ is the original $y$ value corresponding to $x$ value, $\hat{y}_{i}$ is the estimated $y$ value corresponding to $x$ value, and $\bar{y}$ is the mean value of all y values. For the fatigue life prediction case $y_{i}$ is applied cyclic stress, $\hat{y}_{i}$ is the estimated cyclic stress, and $\bar{y}$ is the mean value of all the applied stresses. Furthermore, the accordance of $(x, y)$ data pairs for the correlation can be measured by sample correlation coefficient $r$ as

$r=\frac{\sum_{i=1}^{n} x_{i} y_{i}-\frac{\left(\sum_{i=1}^{n} x_{i}\right)\left(\sum_{i=1}^{n} y_{i}\right)}{n}}{\sqrt{\left(\sum_{i=1}^{n}\left(x_{i}-\bar{x}\right)^{2}\right)\left(\sum_{i=1}^{n}\left(y_{i}-\bar{y}\right)^{2}\right)}}$

with $-1 \leqslant r \leqslant+1 r$ shows how closely $(x, y)$ pairs lie to a straight line. The value of $r$ is expected to be as close as possible to +1 or -1 , depending on whether the slope of the data is positive or negative, respectively [19].

The fatigue life predictions belong to the aforementioned composite materials are given as stress amplitude $\left(\sigma_{\mathrm{a}}\right)$ versus logarithmic fatigue life $(\log N)$ in Figs. $2-5$. In Fig. 2, the fatigue life predictions for $\left[0_{2} / 90_{2}\right]_{S},\left[0 / 90_{4}\right]_{S}$ and $[0 / 45 /-45 / 90]_{S}$ carbon/ epoxy laminates [15] are presented with the experimental data. It is seen that the predictions are in good agreement with the data. However, it seems that the predictions for $\left[0_{2} / 90_{2}\right]_{s}$ and $\left[0 / 90_{4}\right]_{s}$ laminates do not properly fit to the experimental data considering the small $R^{2}$ values $(0.2365$ and 0.3072$)$. This situation does not mean that the correlation is unsatisfactory since the correlation coefficients for the experimental data have low values $(-0.6378$ in Fig. 2(a), and -0.7937 in Fig. 2(b)).

Fig. 3 shows the predictions for the $[0 / 90 / 90 / 0]_{s}$, $[45 / 0 / 0 /-45]_{s}$ and $[45 / 90 /-45 / 0]_{s} \quad$ E-glass/epoxy laminates [16]. It can be noted that all the fatigue life predictions are in a very 
(a)

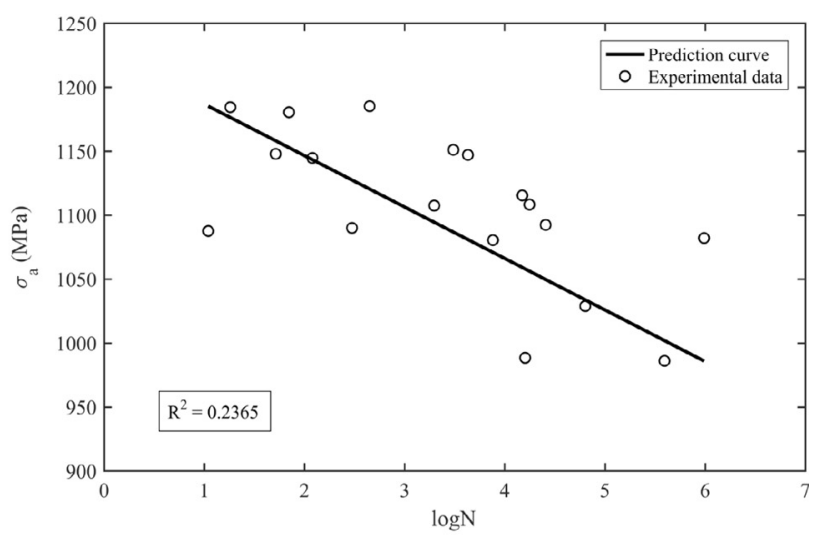

(b)

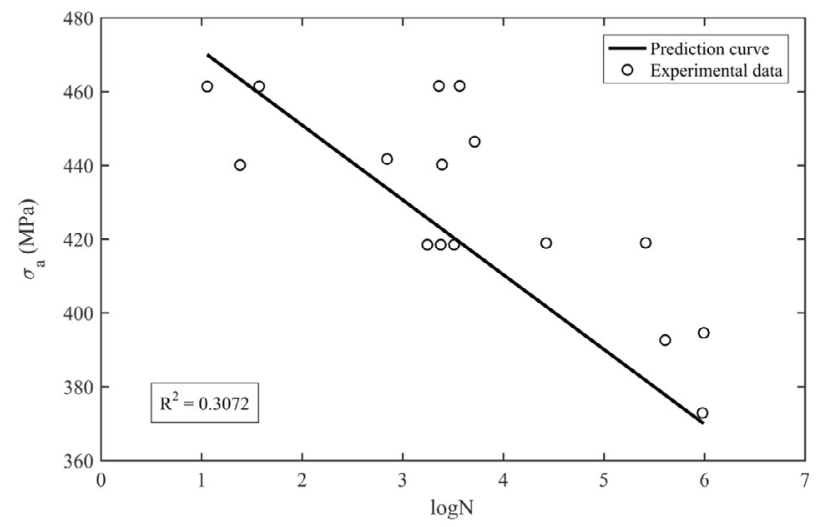

(c)

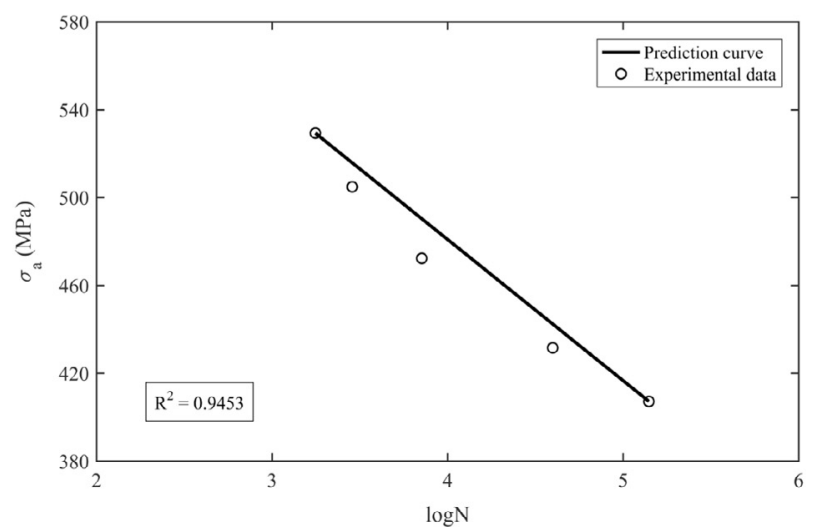

Fig. 2. Fatigue life predictions of (a) $\left[0_{2} / 90_{2}\right]_{s}$, (b) $\left[0 / 90_{4}\right]_{s}$, and (c) $[0 / 45 /-45 / 90]_{s}$ carbon/epoxy laminates.

good agreement. High $R^{2}$ values support the good correlation between experimental data and the predictions of the laminates.

Figs. 4 and 5 show the predictions for $[0 / 90]_{4 s},[0 / 45 / 90 /-45]_{2 s}$ carbon/PEEK [17], and $[30 /-30]_{3},[60 /-60]_{3}$ glass/polyester thermoplastic composite laminates [18], respectively. It is seen that the model predictions are also in good agreement with the experimental data for these thermoplastic composites.

It can be inferred from this correlation study that the FTPF criterion model can properly simulate the fatigue life for a variety of composite materials in multidirectional stacking sequences, and it
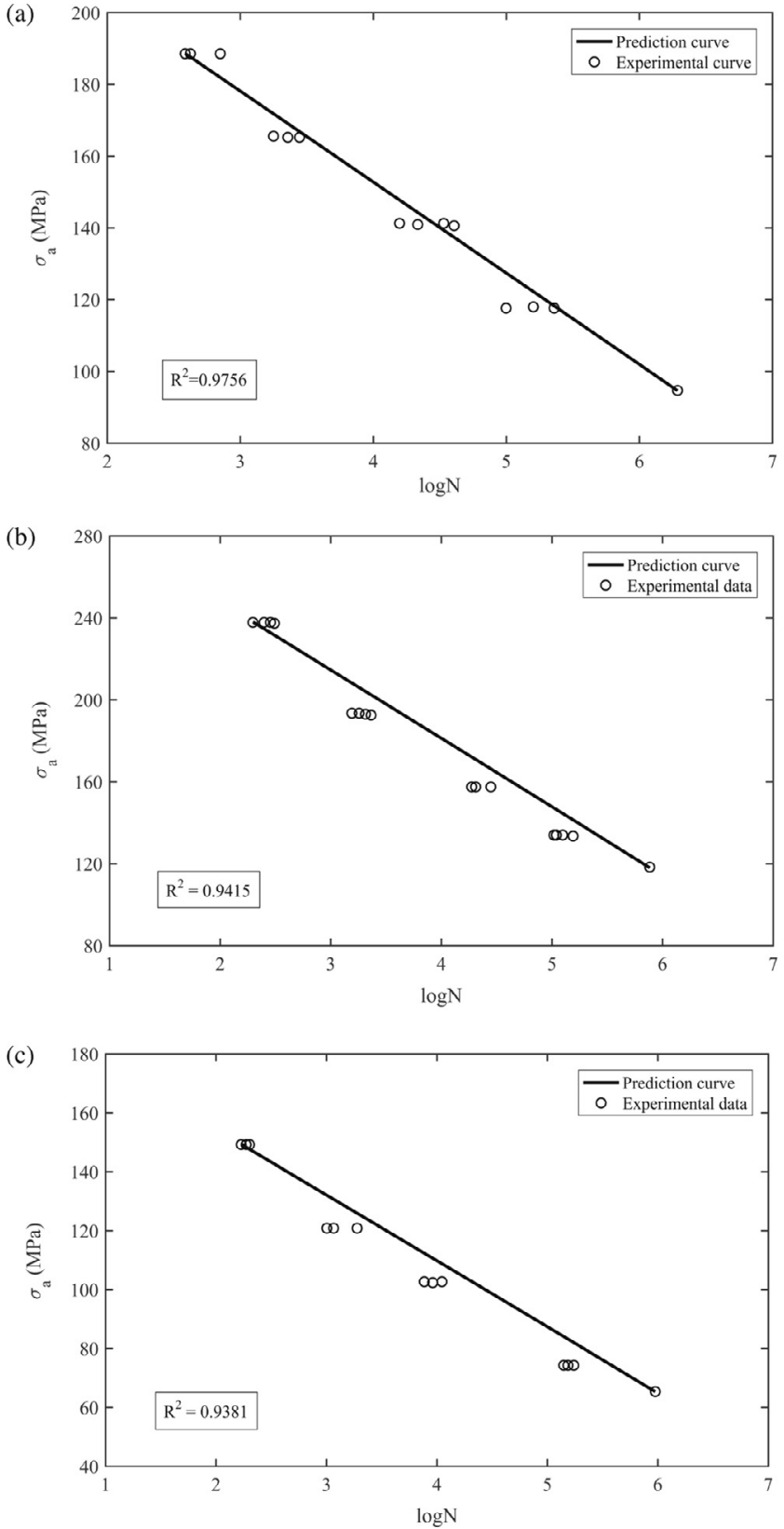

Fig. 3. Fatigue life predictions of (a) $[0 / 90 / 90 / 0]_{s}$, (b) $[45 / 0 / 0 /-45]_{s}$, and (c) $[45 / 90 /-45 / 0]_{s}$ E-glass/epoxy laminates.

is appropriate to be used in the optimum fatigue-resistant design efforts of multidirectional composite plates.

\section{Optimization}

As a modern evolutionary algorithm technique, genetic algorithm (GA) provides important advantages against traditional optimization algorithms, such as robustness to problem complexity and the ability to easily discover global optimum rather than local stationary optima. Nevertheless, standard GA has the disadvantages of slow convergence rates when they work with complicated or time consuming objective functions, being stuck with local optima, and the lack of cooperation between populations. In order to overcome these weak points, some hybrid methods combining two different metaheuristic approaches have been used by the researchers to take advantages of each powerful side of these 

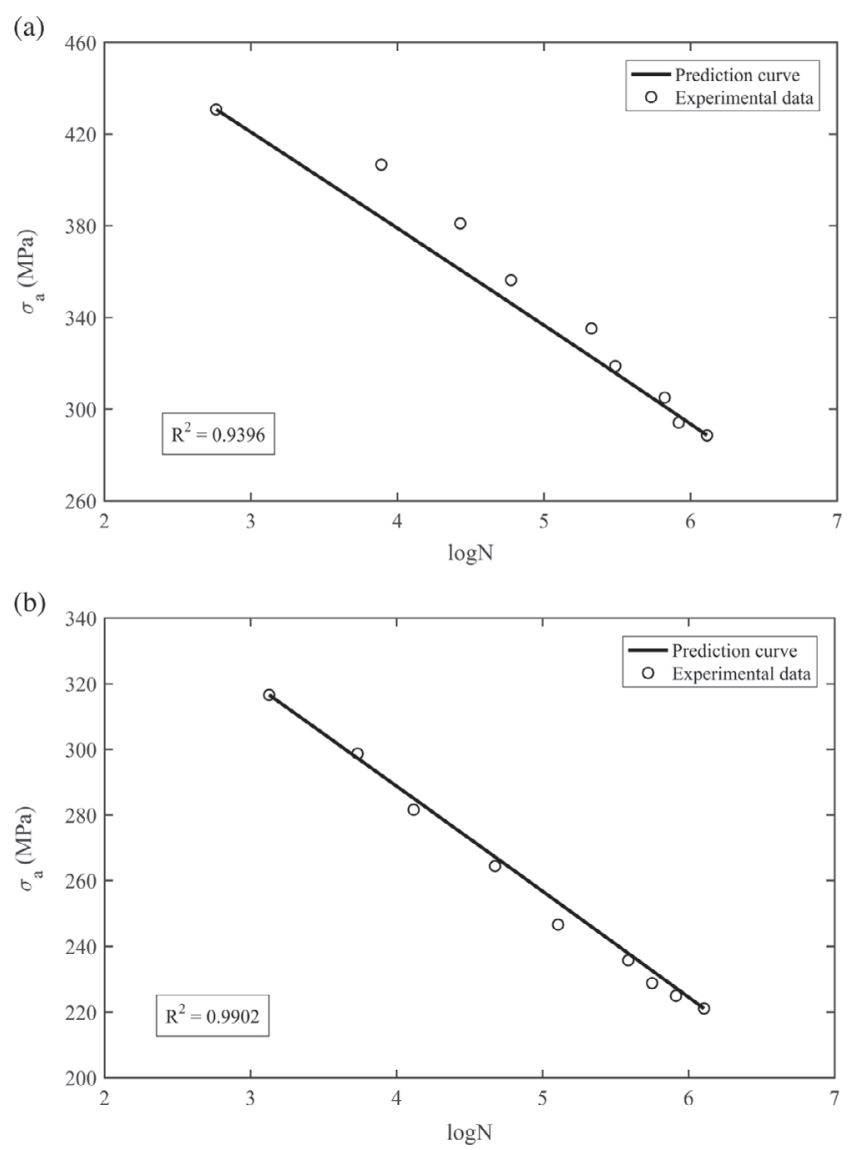

Fig. 4. Fatigue life predictions of (a) $[0 / 90]_{4 s}$, and (b) $[0 / 45 / 90 /-45]_{2 s}$ carbon/PEEK laminates.

approaches $[20,21]$. For this purpose, first, GA is applied to obtain a point close to the global minimum, and then the other chosen algorithm is applied to refine and improve the result obtained by GA, thus, global convergence can be more guaranteed depending on the nature of the problem [22]. Accordingly, in this study, the combination of GA and generalized pattern search algorithm (GPSA) is considered as the hybrid algorithm to achieve a high accuracy rate in our results. In the following sections, brief information about each algorithm is given.

GA is a widely-used heuristic algorithm developed by John Holland [23] in the solution of laminated composite problems. GA utilizes the natural selection process which ends up with the evolution of organisms best adapted to the environment. GA begins its search with a population of random individuals, and the process is carried out by applying operators similar to natural genetic processes, which are called as selection, crossover, mutation and replacement. The process is iterated over many generations until final optimal designs are obtained [24].

GPSA on the other hand is a derivative-free optimization method developed by Torczon [25] for unconstrained optimization of functions and later extended to cover nonlinear constrained optimization problems. As opposed to the traditional local optimization methods that use information about the gradient or partial derivatives to search for an optimal solution, GPSA is a direct search method which finds a sequence of points $x_{i}$ that approach the global optimal point through many iterations. Each iteration consists of two phases: the search phase and the poll phase. In the search phase, the objective function is evaluated at a finite number of points on a mesh to find a new point with a lower objective function value than the best current solution. In the poll phase,
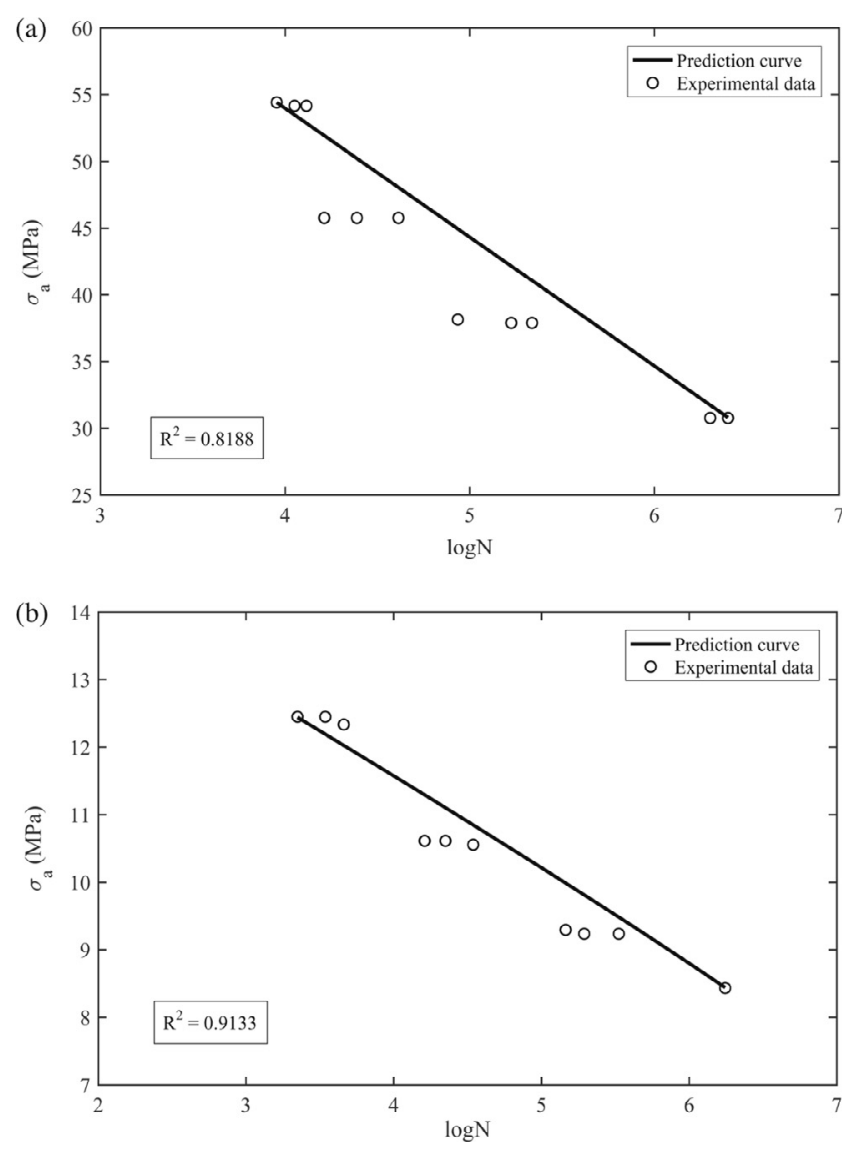

Fig. 5. Fatigue life predictions of (a) $[30 /-30]_{3}$ and (b) $[60 /-60]_{3}$ glass/polyester laminates.

the objective function is evaluated at the neighboring mesh points to see if a lower objective function value can be obtained [26].

The optimization procedure which describes how GPSA works and interacts with GA in the hybrid algorithm is given in Fig. 6 and explained step by step as follows:

Step 1. GA runs until either the maximum number of iterations is reached or there is no improvement in the fitness value.

Step 2. When GA terminates, the reached optimal solution is used as an initial point for GPSA to search.

Step 3. GPSA starts its search with an initial solution $x_{0}$ and an initial mesh size $\Delta_{0}^{m}$.

Step 4. If the search phase satisfies a solution with a lower objective function value than the best current solution, the algorithm stops.

Step 5. If termination criteria not satisfied, the algorithm goes to the poll phase and generates a set of neighboring mesh points $x_{i}^{m}$ by multiplying the current mesh size by each pattern vector $\left\{d_{i}\right\}$. The fixed-direction pattern vectors are used to determine the points to search at each iteration and defined by the independent variables in the objective function; commonly the maximal basis with $2 \mathrm{~N}$ vectors consisting of $N$ positive and $N$ negative vectors, and the minimal basis with $N+1$ vectors.

Step 6. In the polling step at $k$ th iteration, GPSA polls all the mesh points by computing their objective function values $f\left(x_{i}^{m}\right)$ in order to find an improved point.

Step 7. If the poll is successful, which means an improved point is found, the current mesh size is multiplied as $\Delta_{i+1}^{m}=2 \Delta_{i}^{m}$, and the current point is updated by the new mesh size 


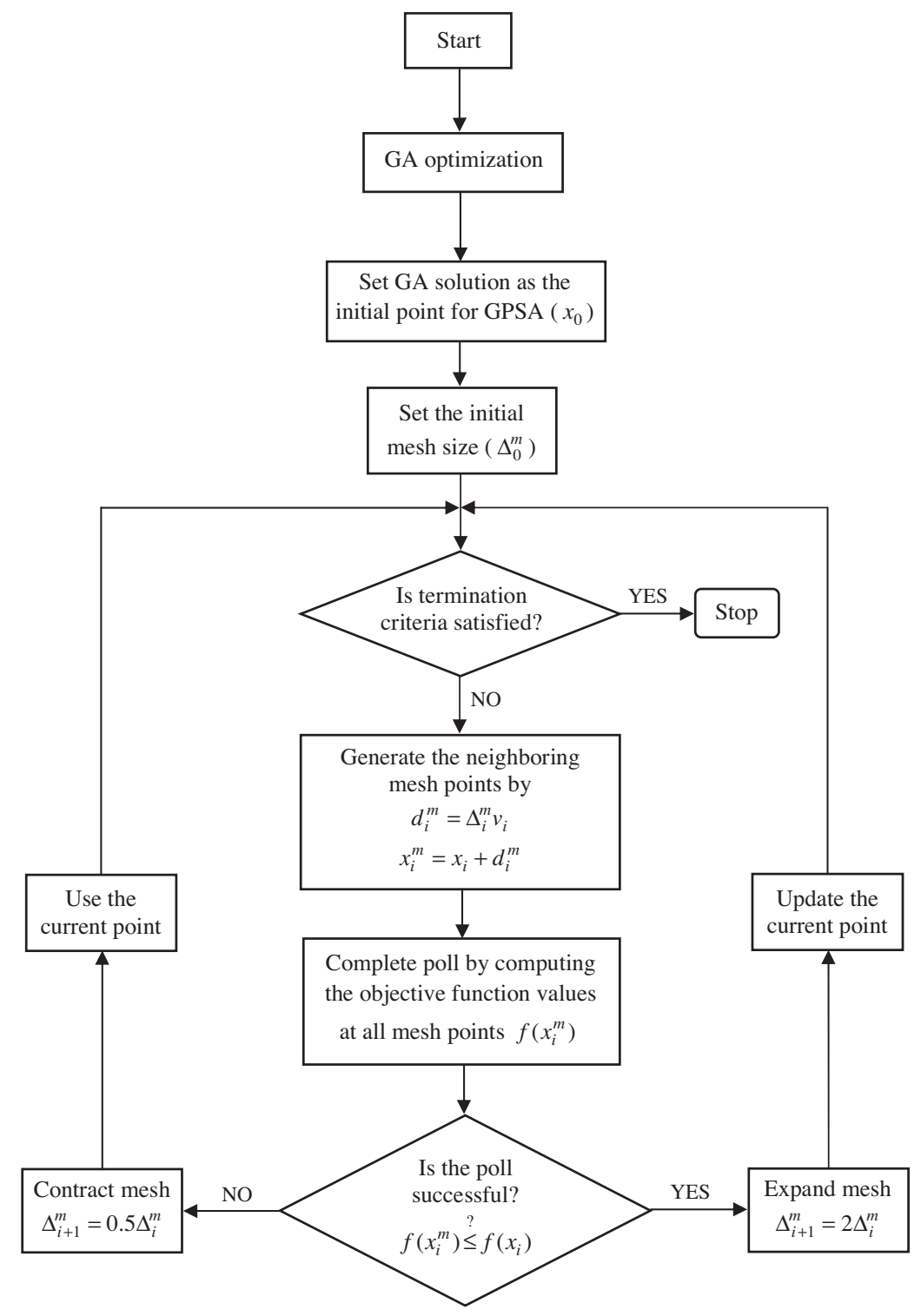

Fig. 6. Flowchart of the hybrid algorithm optimization.

for the next iteration $k+1$. If the polling fails to find an improved point, the mesh size is reduced by $\Delta_{i+1}^{m}=0.5 \Delta_{i}^{m}$, and this current point is used for the next iteration.

This process continues through many iterations until global optimum is reached.

In our study, this hybrid GA-GPSA algorithm is used in fatigue optimization problems. In the literature, different (single or hybrid) approaches have already been used for the modeling of the fatigue life of composite materials. For example, a genetic programming method that finally evolves a computer program is used in [27] for modeling the fatigue life of various laminated composite materials. Also, artificial neural networks based methods are commonly used for fatigue life modeling of different unidirectional and multidirectional laminated composites [28-31]. Furthermore, some hybrid methods are used to predict the fatigue life of glass fiber reinforced composites. For example, a hybrid method combining artificial neural networks and fuzzy logic is used for modeling fatigue behavior of unidirectional glass/epoxy composites in [32]. However, there is not any study in the literature on fatigue life modeling and/or optimization study by hybridization of heuristic algorithms. In this regard, the proposed hybrid GA-GPSA algorithm brings a novel approach to the solution of optimization problems for fatigue life advance of laminated composites.

\section{Problem definition}

In this study, the main objective is to investigate the optimum fiber stacking sequences of the laminated composites for maximum fatigue life. The stacking sequence of the laminate and fatigue life $N$ are determined for each design case in the optimization. The number of distinct laminae $n$ and the thickness of the laminae $t_{0}$ of the laminates are predefined in the design. The orientation angles are considered as the discrete values of $0^{\circ}$, $45^{\circ},-45^{\circ}, 90^{\circ}$ which are conventional in industry. The composite material used in this study is taken from the study of Hashin and Rotem [7]. The laminated composite material is a unidirectional 32-layer E-glass/epoxy. The ply thickness $t_{0}$ is $0.127 \mathrm{~mm}$. Stress ratio $R$ is 0.1 and frequency $v$ is $19 \mathrm{~Hz}$. The material, strength and fatigue properties are presented in Table 1 . We have considered several problems including in-plane cyclic loadings $\mathrm{N}_{\mathrm{xx}}, \mathrm{N}_{\mathrm{yy}}, \mathrm{N}_{\mathrm{xy}}$ 
an initial mesh size of 1 , a mesh expansion factor of 2 , a mesh contraction factor of 0.5 , and a positive basis $2 \mathrm{~N}$ poll method with the options of complete polling and consecutive polling order.

\subsection{Algorithm performance}

A buckling optimization problem previously studied [33,35] was considered as a test problem and solved with the selected options to evaluate the performance of the hybrid algorithm in terms of best stacking sequences giving maximum critical buckling load factor. The results of the hybrid algorithm are compared with the best known results studied by different hybrid algorithms in the literature [36-39]. Table 2 shows the details of composite plate dimensions $a$ and $b$, and in-plane loads $\mathrm{N}_{\mathrm{xx}}$ and $\mathrm{N}_{\mathrm{yy}}$ for the load cases. The optimum critical buckling load factors for all the load cases are compiled from the literature and presented together with the present critical buckling load factor and stacking sequence results in Table 3.

In the table, the critical buckling load factor $\left(\lambda_{c b}\right)$ results are given in British units to provide consistency with the literature.

Table 2

Load cases for test problem.

\begin{tabular}{llllll}
\hline $\begin{array}{l}\text { Load } \\
\text { case }\end{array}$ & $\begin{array}{l}\text { Number } \\
\text { of plies }\end{array}$ & $a(\mathrm{~mm})$ & $b(\mathrm{~mm})$ & $\mathrm{N}_{\mathrm{xx}}(\mathrm{N} / \mathrm{mm})$ & $\mathrm{N}_{\mathrm{yy}}(\mathrm{N} / \mathrm{mm})$ \\
\hline 1 & 48 & 508 & 127 & 17.5 & 2.2 \\
2 & 48 & 508 & 127 & 17.5 & 4.4 \\
3 & 48 & 508 & 127 & 17.5 & 8.8 \\
4 & 64 & 508 & 254 & 17.5 & 17.5 \\
\hline
\end{tabular}

Table 3

Performance results of the hybrid algorithm.

\begin{tabular}{llll}
\hline $\begin{array}{l}\text { Load } \\
\text { case }\end{array}$ & $\begin{array}{l}\lambda_{c b}^{[36-39]} \\
\left(\left[\mathrm{lbf} / \mathrm{in}^{3}\right] /\right.\end{array}$ & $\begin{array}{l}\lambda_{c b}^{\text {present }} \\
\left(\left[\mathrm{lbf} / \mathrm{in}^{3}\right] /\right.\end{array}$ & Stacking sequence ${ }^{\text {present }}$ \\
& $\left.\left[\mathrm{lbf} / \mathrm{in}^{3}\right]\right)$ & $\left.\left[\mathrm{lbf} / \mathrm{in}^{3}\right]\right)$ & \\
\hline 1 & 16120.38 & 20950.55 & {$\left[90_{4} /\left( \pm 45_{2} / 90_{2}\right)_{2} / \pm 45_{3} / 90_{2}\right]_{\mathrm{s}}$} \\
& 16119.48 & 20920.39 & {$\left[90_{2} /\left(90_{2} / \pm 45\right)_{2} / \pm 45_{5} / 90_{2} / \pm 45\right]_{\mathrm{s}}$} \\
& 16087.83 & 20894.53 & {$\left[90_{4} / \pm 45_{3} / 90_{4} / \pm 45_{5}\right]_{\mathrm{s}}$} \\
2 & 13442.04 & 15961.75 & {$\left[90_{4} / \pm 45 /\left(90_{2} / \pm 45_{2}\right)_{2} / \pm 45_{3}\right]_{\mathrm{s}}$} \\
& 13441.28 & 15729.07 & {$\left[90_{2} /\left(90_{2} / \pm 45\right)_{2} / \pm 45_{4} / 90_{2} / \pm 45_{2}\right]_{\mathrm{s}}$} \\
3 & 13435.94 & 15512.55 & {$\left[90_{4} / \pm 45_{2} / 90_{2} / \pm 45_{7}\right]_{\mathrm{s}}$} \\
& 10003.53 & 10591.61 & {$\left[90_{2} / \pm 45 / 90_{2} /\left(90_{2} / \pm 45\right)_{2} / \pm 45_{4} / 90_{2}\right]_{\mathrm{s}}$} \\
& 10002.95 & 10460.19 & {$\left[90_{2} /\left(90_{2} / \pm 45\right)_{2} / \pm 45_{5} / 90_{2} / \pm 45\right]_{\mathrm{s}}$} \\
4 & 9999.45 & 10343.85 & {$\left[\left(90_{2} / \pm 45\right)_{3} / \pm 45 / 90_{2} / \pm 45_{4}\right]_{\mathrm{s}}$} \\
& $3973.01^{*}$ & 3973.01 & {$\left[90_{10} / \pm 45 / 90_{2} / \pm 45_{7} / 90_{2} / \pm 45\right]_{\mathrm{s}}$} \\
& 3973.00 & 3973.00 & {$\left[90_{4} /\left(90_{2} / \pm 45\right)_{3} / 90_{8} / \pm 45 / 90_{6}\right]_{s}$} \\
\hline
\end{tabular}

" The optimum values calculated from the reference stacking sequences.
Ply contiguity constraint for stacking sequences is applied to the first three load cases. Besides, stacking sequences are subjected to symmetry and balance constraints. In order to provide an average quality of solutions, each load case is performed 100 times with different starting points. It can be seen from the table that $\lambda_{c b}$ values for the test problem are found superior to the results given in the literature for load cases 1,2 and 3 . In load case 4, the same results are obtained for the first and second optima. $\lambda_{c b}$ values denoted with asterisk sign are the real optimum values calculated from the stacking sequence designs given by the related references. It can be noted that $\lambda_{c b}$ values found by [36-39] are possibly misrepresented due to round of error in their optimization procedures. This test study implies that the hybrid algorithm shows very good performance in searching the design space for laminated composite optimization, and has capability to yield the best possible results for the fatigue optimization studies.

\subsection{Fatigue optimization}

In the fatigue optimization study, multidirectional laminate derivations are produced to increase the fatigue life theoretically using the FTPF model and hybrid algorithm. It is obvious that the optimum stacking sequences giving maximum fatigue lives require a validation supporting the proposed fatigue optimization strategy. In this regard, a pre-optimization study is performed to justify the theoretical derivation procedure using experimental data from the literature. The prediction and optimization procedures are applied to different multidirectional composite laminates $[15,16,40]$. For each laminate, first, estimated fatigue life is determined. Then, the optimum laminate configurations to be replaced with the tested laminate are investigated and the stacking sequences with increased fatigue lives are obtained. The results are presented with the stacking sequences and experimental fatigue lives of the reference materials in Table 4. In the table, first two optimum results for each case are shown, and the fatigue life values are given as logarithmic.

As seen in Table 4, the predicted fatigue life values are found to be very close to the experimental fatigue life values, especially for E-glass/epoxy laminates [16]. The optimization results show that longer fatigue lives can be obtained with different stacking sequences of the laminates. For example, while an approximated fatigue life of 5.97 is reached experimentally and predictively for the $[45 / 90 /-45 / 0]_{s}$ sequence [15], fatigue lives of 6.9939 and 6.4057 can be achieved by $\left[45 / 0_{3}\right]_{s}$ and $[45 / 0]_{2 s}$ sequences, respectively. Considering that the fatigue lives of the laminates in $[15,16,40]$ are accurately predicted by the FTPF model, it can be concluded that the optimum results obtained theoretically will be acceptable.

Table 4

Fatigue life prediction and optimization using different experimental data from the literature.

\begin{tabular}{|c|c|c|c|c|}
\hline Stacking sequence $[15,16,40]$ & Experimental fatigue life $[15,16,40]$ & Predicted fatigue life & Optimum stacking sequence & Maximum fatigue life \\
\hline \multirow[t]{2}{*}[0/90/90/0]{$_{S}$} & 6.2871 & 6.2809 & {$\left[0_{3} / 90\right]_{S}$} & 7.4888 \\
\hline & & & {$[0 / 90]_{2 s}$} & 6.2809 \\
\hline \multirow[t]{2}{*}[45/0/0/-45]{$_{s}$} & 5.8845 & 5.8831 & {$\left[0_{3} / 45\right]_{S}$} & 7.9712 \\
\hline & & & {$[0 / 45]_{2 s}$} & 7.8077 \\
\hline \multirow[t]{2}{*}[45/90/-45/0]{$_{s}$} & 5.9772 & 5.9745 & {$\left[45 / 0_{3}\right]_{S}$} & 6.9939 \\
\hline & & & {$[45 / 0]_{2 s}$} & 6.4057 \\
\hline \multirow[t]{2}{*}[0/90_{4}]{$_{s}$} & 5.9804 & 5.9939 & {$\left[0_{4} / 90\right]_{S}$} & 7.4419 \\
\hline & & & {$\left[0_{4} / 45\right]_{S}$} & 6.7113 \\
\hline \multirow[t]{2}{*}[0_{2}/90_{2}]{$_{s}$} & 5.5941 & 5.9900 & {$\left[0_{3} / 45\right]_{S}$} & 5.9974 \\
\hline & & & {$[0 / 90]_{2 s}$} & 5.9900 \\
\hline \multirow[t]{2}{*}[0/90]{$_{4 s}$} & 6.1121 & 6.1299 & {$\left[0_{3} / 90\right]_{2 s}$} & 6.5118 \\
\hline & & & {$\left[0_{2} / 90_{2}\right]_{2 s}$} & 6.1299 \\
\hline \multirow[t]{2}{*}[0/\pm45/90]{$_{2 s}$} & 6.0486 & 6.3835 & {$\left[0_{3} / 90\right]_{2 s}$} & 6.4213 \\
\hline & & & {$[0 / 90 / \pm 45]_{2 s}$} & 6.3835 \\
\hline
\end{tabular}


The fatigue optimization study consists of two parts. As the main part, optimization problems are solved using the discrete fiber angles. As the complementary part, an optimization study is performed using integer fiber angle values between $-90^{\circ}$ and $90^{\circ}$ for some selected design cases and the results are compared with conventional stacking sequence designs containing the discrete fiber angles. In order to increase the efficiency and reliability of the algorithm, at least 50 independent searches are performed for each case. Different load levels and combinations which allow feasible designs are investigated. The optimum stacking sequences of laminates, the corresponding fatigue lives, and the number of global optima found for various in-plane cyclic loads $\left(\mathrm{N}_{\mathrm{xx}} / \mathrm{N}_{\mathrm{yy}} /\right.$ $\mathrm{N}_{\mathrm{xy}}$ ) are presented in Tables 5-7. Since multiple global optima exist in many loading cases, only one stacking sequence is shown for each loading in the tables. Failure indexes of the laminates determined according to HR failure criterion are indicated separately as $F I_{\text {fiber }}$ and $F I_{\text {matrix }}$ for fiber and matrix in the tables. Finally, the results of the comparison study between the optimization with discrete fiber angles and the optimization with integer fiber angles are given in Table 8 .

Table 5 shows the results for only tension cyclic loads. As the results indicate, the fatigue life is found to be sensitive to the level of stress. For each $\mathrm{N}_{\mathrm{xx}}$ loading levels of $5,7.5,10\left(\times 10^{2} \mathrm{~N} / \mathrm{mm}\right)$, fatigue life of the optimum designs decreases with the increase of $\mathrm{N}_{\mathrm{yy}}$ loading as may be expected. Maximum fatigue lives of the optimum designs are achieved between $10^{6}$ and $10^{8}$ cycles. However, fatigue lives in the range of $10^{3}-10^{4}$ cycles are able to be reached for the designs of 5/10/0,10/5/0 and 10/7.5/0 critical loadings. Unpredictably, in the design cases for 7.5/2.5/0 and 10/2.5/0 loadings, the designs with more fatigue life are obtained compared to the 7.5/0/0 and 10/0/0 loading cases, respectively. It is also noted that the same fatigue lives are obtained with different stacking sequence designs for 5/10/0-10/5/0 and 7.5/10/0-10/7.5/0 loadings. Furthermore, failure indexes indicate that all the laminate configurations are reliable against static failure.

Table 6 shows the effect of the existence of shear stress on the optimum designs. It is seen that fatigue life dramatically decreases in the presence of shear load. For instance, the fatigue lives of the designs obtained for $5 / 0 / 2.5,5 / 2.5 / 2.5$ and $5 / 5 / 2.5$ loadings are less than the ones for 5/0/0,5/2.5/0 and 5/5/0 loadings in Table 5 . In the same manner, a considerable decrease occurs when the applied shear load is increased. For instance, the designs found for $5 / 0 / 5$, $5 / 2.5 / 5$ and $5 / 5 / 5$ loadings have quite less fatigue lives than those for $5 / 0 / 2.5,5 / 2.5 / 2.5$ and 5/5/2.5 loadings. The increase of $N_{x x}$ also decreases the fatigue life of composites. This decrease can be seen in $2.5 / 0 / 2.5,5 / 0 / 2.5,7.5 / 0 / 2.5$ and $10 / 0 / 2.5$ loadings. It is also noted that for $0 / 5 / 2.5-5 / 0 / 2.5$ and $0 / 7.5 / 2.5-7.5 / 0 / 2.5$ loadings different stacking sequences are obtained with the same fatigue life values. All the laminates are safe against static loading even if they have short fatigue lives. In general, it can be said that fatigue life dramatically changes and mostly decreases according to the shear load level and its applied combination.

In Table 7, the optimum results for tension-compression (T-C) and compression-compression (C-C) loadings are given. It is seen that the optimum stacking sequence designs for T-C loading yield less fatigue life than the previous designs for tension-tension ( $\mathrm{T}$ $\mathrm{T}$ ) loading. For instance, the optimum design found for $5 /-2.5 / 0$ loading has a fatigue life of $2.833 \times 10^{7}$ cycles whereas it is $5.503 \times 10^{7}$ cycles for $5 / 2.5 / 0$ loading. However, it is found that the stacking sequence designs for $\mathrm{C}-\mathrm{C}$ loading have longer fatigue

Table 5

Optimum stacking sequence designs and the corresponding fatigue lives for various in-plane tension-tension cyclic loads.

\begin{tabular}{|c|c|c|c|c|c|}
\hline Loading $\mathrm{N}_{\mathrm{xx}} / \mathrm{N}_{\mathrm{yy}} / \mathrm{N}_{\mathrm{xy}}\left(\times 10^{2} \mathrm{~N} / \mathrm{mm}\right)$ & Stacking sequence & No. of global optima & Fatigue life (cycles) & $F I_{\text {fiber }}$ & $F I_{\text {matrix }}$ \\
\hline $5 / 0 / 0$ & {$\left[0_{4} / 90_{2} / 0_{4} / 90_{2} / 0_{4}\right]_{S}$} & 8 & $1.283 \times 10^{8}$ & 0.0170 & 0.1009 \\
\hline $5 / 2.5 / 0$ & {$\left[0_{4} / \pm 45_{3} / 0_{2} / \pm 45_{2}\right]_{s}$} & 25 & $5.503 \times 10^{7}$ & 0.1420 & 0.1849 \\
\hline $5 / 5 / 0$ & {$\left[\left(0_{2} / 90_{2}\right)_{2} / 90_{2} / 0_{4} / 90_{2}\right]_{s}$} & 20 & $4.660 \times 10^{6}$ & 0.1858 & 0.3350 \\
\hline $5 / 7.5 / 0$ & {$\left[90_{2} / 0_{2} /\left(0_{2} / 90_{4}\right)_{2}\right]_{s}$} & 32 & $2.979 \times 10^{5}$ & 0.2374 & 0.5387 \\
\hline $5 / 10 / 0$ & {$\left[90_{2} / \pm 45 / 90_{4} / \pm 45_{4}\right]_{s}$} & 22 & $3.175 \times 10^{4}$ & 0.2839 & 0.7398 \\
\hline $7.5 / 0 / 0$ & {$\left[0_{4} / 90_{2} / 0_{4} / \pm 45 / 0_{4}\right]_{S}$} & 10 & $3.229 \times 10^{6}$ & 0.1900 & 0.1953 \\
\hline $7.5 / 2.5 / 0$ & {$\left[0_{2} /\left(0_{2} / \pm 45_{2}\right)_{2} / 0_{2}\right]_{S}$} & 23 & $6.667 \times 10^{6}$ & 0.2067 & 0.2794 \\
\hline $7.5 / 5 / 0$ & {$\left[\left(90_{2} / 0_{2}\right)_{2} / 0_{2} / 90_{2} / 0_{4}\right]_{s}$} & 29 & $2.979 \times 10^{5}$ & 0.2374 & 0.5387 \\
\hline $7.5 / 7.5 / 0$ & {$\left[ \pm 45 / 0_{2} / \pm 45 / 90_{2} / 0_{2} / 90_{2} / \pm 45_{2}\right]_{s}$} & 67 & $2.826 \times 10^{4}$ & 0.2787 & 0.7538 \\
\hline $7.5 / 10 / 0$ & {$\left[ \pm 45_{5} / 90_{2} / \pm 45_{2}\right]_{s}$} & 8 & $2.058 \times 10^{3}$ & 0.3823 & 0.9882 \\
\hline $10 / 0 / 0$ & {$\left[0_{4} / \pm 45 / 0_{4} / 90_{2} / 0_{4}\right]_{s}$} & 3 & $2.224 \times 10^{5}$ & 0.2533 & 0.3472 \\
\hline $10 / 2.5 / 0$ & {$\left[\left(0_{4} / \pm 45\right)_{2} / 0_{2} / \pm 45\right]_{S}$} & 25 & $7.752 \times 10^{5}$ & 0.2503 & 0.4284 \\
\hline $10 / 5 / 0$ & {$\left[\left( \pm 45_{2} / 0_{2}\right)_{2} / 0_{2} / \pm 45\right]_{s}$} & 22 & $3.175 \times 10^{4}$ & 0.2839 & 0.7398 \\
\hline $10 / 7.5 / 0$ & {$\left[ \pm 45_{6} / 0_{2} / \pm 45\right]_{s}$} & 6 & $2.058 \times 10^{3}$ & 0.3823 & 0.9882 \\
\hline
\end{tabular}

Table 6

Optimum stacking sequence designs and the corresponding fatigue lives for various in-plane tension and shear cyclic loads.

\begin{tabular}{|c|c|c|c|c|c|}
\hline Loading $\mathrm{N}_{\mathrm{xx}} / \mathrm{N}_{\mathrm{yy}} / \mathrm{N}_{\mathrm{xy}}\left(\times 10^{2} \mathrm{~N} / \mathrm{mm}\right)$ & Stacking sequence & No. of global optima & Fatigue life (cycles) & $F I_{\text {fiber }}$ & $F I_{\text {matrix }}$ \\
\hline $0 / 0 / 5$ & {$\left[ \pm 45_{8}\right]_{s}$} & 1 & $3.987 \times 10^{6}$ & 0.1912 & 0.1196 \\
\hline $0 / 5 / 2.5$ & {$\left[ \pm 45_{3} / 90_{4} / \pm 45 / 90_{4}\right]_{s}$} & 22 & $4.344 \times 10^{4}$ & 0.1990 & 0.1940 \\
\hline $0 / 7.5 / 2.5$ & {$\left[90_{4} / \pm 45 / 90_{4} / \pm 45_{2} / 90_{2}\right]_{s}$} & 18 & $1.938 \times 10^{3}$ & 0.2580 & 0.3214 \\
\hline $2.5 / 0 / 2.5$ & {$\left[ \pm 45 / 0_{2} / \pm 45_{4} / 0_{2} / \pm 45\right]_{s}$} & 22 & $6.738 \times 10^{5}$ & 0.1592 & 0.1079 \\
\hline $2.5 / 2.5 / 2.5$ & {$\left[ \pm 45_{8}\right]_{s}$} & 1 & $1.023 \times 10^{7}$ & 0.1885 & 0.2137 \\
\hline $5 / 0 / 2.5$ & {$\left[\left( \pm 45 / 0_{2}\right)_{4}\right]_{s}$} & 19 & $4.344 \times 10^{4}$ & 0.1990 & 0.1940 \\
\hline $5 / 2.5 / 2.5$ & {$\left[\left(0_{2} / \pm 45\right)_{2} / \pm 45_{4}\right]_{s}$} & 14 & $2.642 \times 10^{5}$ & 0.2491 & 0.3838 \\
\hline $5 / 5 / 2.5$ & {$\left[ \pm 45_{8}\right]_{S}$} & 1 & $8.551 \times 10^{4}$ & 0.2814 & 0.5651 \\
\hline $5 / 0 / 5$ & {$\left[0_{2} / \pm 45_{2} / 0_{4} / \pm 45_{3}\right]_{s}$} & 21 & 46 & 0.3157 & 0.4352 \\
\hline $5 / 2.5 / 5$ & {$\left[ \pm 45 / 0_{2} / \pm 45_{5} / 0_{2}\right]_{s}$} & 11 & 932 & 0.3734 & 0.7102 \\
\hline $5 / 5 / 5$ & {$\left[ \pm 45_{8}\right]_{s}$} & 1 & 962 & 0.3770 & 0.8550 \\
\hline $7.5 / 0 / 2.5$ & {$\left[0_{2} / \pm 45 / 0_{2} /\left(0_{2} / \pm 45\right)_{2} / 0_{2}\right]_{s}$} & 19 & $1.938 \times 10^{3}$ & 0.2580 & 0.3214 \\
\hline $7.5 / 2.5 / 2.5$ & {$\left[ \pm 45_{4} / 0_{2} / \pm 45 / 0_{4}\right]_{s}$} & 23 & $1.090 \times 10^{4}$ & 0.2959 & 0.5538 \\
\hline $10 / 0 / 2.5$ & {$\left[\left(0_{4} / \pm 45\right)_{2} / 0_{4}\right]_{S}$} & 14 & 73 & 0.3565 & 0.5709 \\
\hline
\end{tabular}


Table 7

Optimum stacking sequence designs and the corresponding fatigue lives for various in-plane tension and compression cyclic loads.

\begin{tabular}{|c|c|c|c|c|c|}
\hline Loading $\mathrm{N}_{\mathrm{xx}} / \mathrm{N}_{\mathrm{yy}} / \mathrm{N}_{\mathrm{xy}}\left(\times 10^{2} \mathrm{~N} / \mathrm{mm}\right)$ & Stacking sequence & No. of global optima & Fatigue life (cycles) & $F I_{\text {fiber }}$ & $F I_{\text {matrix }}$ \\
\hline $5 /-2.5 / 0$ & {$\left[\left(0_{2} / 90_{2}\right)_{2} / 0_{4} / 90_{2} / 0_{2}\right]_{s}$} & 27 & $2.833 \times 10^{7}$ & 0.1552 & 0.0903 \\
\hline $5 /-5 / 0$ & {$\left[90_{2} /\left(0_{2} / 90_{2}\right)_{2} / 90_{2} / 0_{4}\right]_{s}$} & 36 & $3.987 \times 10^{6}$ & 0.1912 & 0.1196 \\
\hline $5 /-7.5 / 0$ & {$\left[90_{4} /\left(0_{2} / 90_{2}\right)_{2} / 90_{2} / 0_{2}\right]_{s}$} & 20 & $2.709 \times 10^{5}$ & 0.2479 & 0.2099 \\
\hline $5 /-10 / 0$ & {$\left[90_{4} / 0_{4} / 90_{4} / 0_{2} / 90_{2}\right]_{s}$} & 22 & $1.573 \times 10^{4}$ & 0.3103 & 0.3611 \\
\hline$-5 /-5 / 0$ & {$\left[90_{4} / 0_{2} / 90_{2} / 0_{4} / 90_{2} / 0_{2}\right]_{s}$} & 14 & $4.660 \times 10^{6}$ & 0.1858 & 0.3350 \\
\hline $7.5 /-2.5 / 0$ & {$\left[\left(0_{4} / 90_{2}\right)_{2} / 0_{4}\right]_{S}$} & 23 & $3.566 \times 10^{6}$ & 0.1963 & 0.1349 \\
\hline $7.5 /-5 / 0$ & {$\left[0_{2} /\left(0_{2} / 90_{2}\right)_{2} / 0_{4} / 90_{2}\right]_{S}$} & 23 & $2.709 \times 10^{5}$ & 0.2479 & 0.2099 \\
\hline $10 /-2.5 / 0$ & {$\left[\left(0_{4} / 90_{2}\right)_{2} / 0_{4}\right]_{S}$} & 18 & $2.009 \times 10^{5}$ & 0.2615 & 0.2767 \\
\hline $10 /-5 / 0$ & {$\left[0_{4} / 90_{4} / 0_{4} / 90_{2} / 0_{2}\right]_{s}$} & 23 & $1.573 \times 10^{4}$ & 0.3103 & 0.3611 \\
\hline $10 /-7.5 / 0$ & {$\left[0_{2} /\left(0_{2} / 90_{2}\right)_{3} / 0_{2}\right]_{S}$} & 22 & 751 & 0.3710 & 0.5043 \\
\hline $10 /-10 / 0$ & {$\left[0_{4} /\left(0_{2} / 90_{4}\right)_{2}\right]_{s}$} & 27 & 311 & 0.3824 & 0.4784 \\
\hline$-10 /-7.5 / 0$ & {$\left[0_{2} / \pm 45_{7}\right]_{s}$} & 2 & $2.058 \times 10^{3}$ & 0.3823 & 0.9882 \\
\hline
\end{tabular}

Table 8

Comparison of conventional (Con.) and non-conventional (Non-con.) fiber angle optimizations for various in-plane cyclic loadings.

\begin{tabular}{|c|c|c|c|}
\hline Loading $\mathrm{N}_{\mathrm{xx}} / \mathrm{N}_{\mathrm{yy}} / \mathrm{N}_{\mathrm{xy}}\left(\times 10^{2} \mathrm{~N} / \mathrm{mm}\right)$ & Angle type & Stacking sequence & Fatigue life (cycles) \\
\hline \multirow[t]{2}{*}{$5 / 0 / 0$} & Con. & {$\left[0_{4} / 90_{2} / 0_{4} / 90_{2} / 0_{4}\right]_{s}$} & $1.283 \times 10^{8}$ \\
\hline & Non-con. & {$\left[0_{16}\right]_{S}$} & $4.704 \times 10^{8}$ \\
\hline \multirow[t]{2}{*}{$5 / 2.5 / 0$} & Con. & {$\left[0_{4} / \pm 45_{3} / 0_{2} / \pm 45_{2}\right]_{s}$} & $5.503 \times 10^{7}$ \\
\hline & Non-con. & {$\left[ \pm 35_{8}\right]_{s}$} & $6.373 \times 10^{7}$ \\
\hline \multirow[t]{2}{*}{$5 / 5 / 0$} & Con. & {$\left[\left(0_{2} / 90_{2}\right)_{2} / 90_{2} / 0_{4} / 90_{2}\right]_{s}$} & $4.660 \times 10^{6}$ \\
\hline & Non-con. & {$\left[0_{2} / \pm 55 / \pm 63 / \pm 12 / 90_{2} / \pm 27 / \pm 35 / \pm 78\right]_{s}$} & $4.660 \times 10^{6}$ \\
\hline \multirow[t]{2}{*}{$5 / 7.5 / 0$} & Con. & {$\left[90_{2} / 0_{2} /\left(0_{2} / 90_{4}\right)_{2}\right]_{S}$} & $2.979 \times 10^{5}$ \\
\hline & Non-con. & {$\left[ \pm 51_{8}\right]_{s}$} & $4.959 \times 10^{5}$ \\
\hline \multirow[t]{2}{*}{$5 / 10 / 0$} & Con. & {$\left[90_{2} / \pm 45 / 90_{4} / \pm 45_{4}\right]_{s}$} & $3.175 \times 10^{4}$ \\
\hline & Non-con. & {$[ \pm 55]_{S}$} & $8.230 \times 10^{4}$ \\
\hline \multirow[t]{2}{*}{$5 / 0 / 2.5$} & Con. & {$\left[\left( \pm 45 / 0_{2}\right)_{4}\right]_{s}$} & $4.344 \times 10^{4}$ \\
\hline & Non-con. & {$\left[ \pm 49 / 0_{2} / \pm 49_{2} / 0_{2} / \pm 49 / 0_{4}\right]_{s}$} & $6.946 \times 10^{4}$ \\
\hline \multirow[t]{2}{*}{$5 / 2.5 / 2.5$} & Con. & {$\left[\left(0_{2} / \pm 45\right)_{2} / \pm 45_{4}\right]_{s}$} & $2.642 \times 10^{5}$ \\
\hline & Non-con. & {$\left[ \pm 35_{8}\right]_{s}$} & $6.311 \times 10^{5}$ \\
\hline \multirow[t]{2}{*}{$5 / 5 / 2.5$} & Con. & {$\left[ \pm 45_{8}\right]_{s}$} & $8.551 \times 10^{4}$ \\
\hline & Non-con. & {$\left[ \pm 45_{8}\right]_{s}$} & $8.551 \times 10^{4}$ \\
\hline \multirow[t]{2}{*}{$5 / 2.5 / 5$} & Con. & {$\left[ \pm 45 / 0_{2} / \pm 45_{5} / 0_{2}\right]_{s}$} & 932 \\
\hline & Non-con. & {$\left[ \pm 36_{8}\right]_{S}$} & 3243 \\
\hline \multirow[t]{2}{*}{$5 /-2.5 / 0$} & Con. & {$\left[\left(0_{2} / 90_{2}\right)_{2} / 0_{4} / 90_{2} / 0_{2}\right]_{S}$} & $2.833 \times 10^{7}$ \\
\hline & Non-con. & {$\left[0_{2} / 90_{2} / 0_{4} /\left(90_{2} / 0_{2}\right)_{2}\right]_{s}$} & $2.833 \times 10^{7}$ \\
\hline \multirow[t]{2}{*}{$5 /-7.5 / 0$} & Con. & {$\left[90_{4} /\left(0_{2} / 90_{2}\right)_{2} / 90_{2} / 0_{2}\right]_{s}$} & $2.709 \times 10^{5}$ \\
\hline & Non-con. & {$\left[\left(90_{2} / 0_{2}\right)_{3} / 90_{4}\right]_{S}$} & $2.709 \times 10^{5}$ \\
\hline \multirow[t]{2}{*}{$7.5 / 2.5 / 0$} & Con. & {$\left[0_{2} /\left(0_{2} / \pm 45_{2}\right)_{2} / 0_{2}\right]_{s}$} & $6.667 \times 10^{6}$ \\
\hline & Non-con. & {$\left[ \pm 29_{8}\right]_{s}$} & $1.580 \times 10^{7}$ \\
\hline \multirow[t]{2}{*}{$7.5 / 5 / 0$} & Con. & {$\left[\left(90_{2} / 0_{2}\right)_{2} / 0_{2} / 90_{2} / 0_{4}\right]_{S}$} & $2.979 \times 10^{5}$ \\
\hline & Non-con. & {$\left[ \pm 39_{8}\right]_{s}$} & $4.959 \times 10^{5}$ \\
\hline \multirow[t]{2}{*}{$10 / 2.5 / 0$} & Con. & {$\left[\left(0_{4} / \pm 45\right)_{2} / 0_{2} / \pm 45\right]_{s}$} & $7.752 \times 10^{5}$ \\
\hline & Non-con. & {$\left[ \pm 26_{8}\right]_{S}$} & $3.953 \times 10^{6}$ \\
\hline
\end{tabular}

lives than the designs for T-C loading. For instance, the design for $-5 /-5 / 0$ loading has $16.88 \%$ higher fatigue life than the design for 5/-5/0 loading. Also, when C-C loading cases are compared to $\mathrm{T}$-T loading cases, it is seen that the same fatigue lives are obtained in different stacking sequences (e.g., $-5 /-5 / 0-5 / 5 / 0$ and $-10 /-7$. $5 / 0-10 / 7.5 / 0)$. This is due to the assumption that tension fatigue failure stress equals to compression fatigue failure stress in the FTPF prediction model as stated earlier.

Table 8 shows the comparison results of the optimization using conventional fiber angles (Con.) and the optimization using nonconventional fiber angles (Non-con.) for selected in-plane cyclic loadings. Stacking sequences and fatigue lives corresponds to related loadings and angle types are given in the table. Optimum results obtained by non-conventional angles are superior or at least comparable to the results obtained by conventional angles. For example, in 5/2.5/2.5 loading, $\left[ \pm 35_{8}\right]_{s}$ design with a fatigue life of $6.311 \times 10^{5}$ cycles is found by non-conventional lamination while $\left[\left(0_{2} / \pm 45\right)_{2} / \pm 45_{4}\right]_{s}$ design with a fatigue life of $2.642 \times 10^{5}$ cycles is found by conventional lamination. This corresponds to an increase of $58.14 \%$ in fatigue life. As in 10/2.5/0 load- ing case, the increase in fatigue life can even be up to $80.39 \%$. Nevertheless, in $5 / 5 / 0,5 / 5 / 2.5,5 /-2.5 / 0$ and $5 /-7.5 / 0$ loading cases, fatigue lives are the same values even if their stacking sequences are different.

As the fatigue optimization results show in general, fatigue life of composites changes dramatically according to type of loading, loading combination and level of stress. Most of the designs are obtained within fatigue life range of $10^{5}-10^{7}$. However, in design cases of high loading, fatigue life can only be increased to $10^{2}$ and $10^{3}$ cycle levels. Especially in the presence of shear and compressive loads, fatigue life significantly decreases. These unsatisfactory results indicate the most critical cases that restrict to develop reasonable fatigue-resistant designs.

\section{Conclusion}

In this study, a procedure is proposed to find the optimum fiber orientations of laminated composites giving maximum fatigue life. For this purpose, a fatigue life prediction model termed as Failure Tensor Polynomial in Fatigue (FTPF) is used to determine the fati- 
gue lives of the laminates. An experimental correlation is performed by using experimental data from the literature to examine the prediction capability of the model. A hybrid algorithm composed of GA and GPSA is used as search algorithm in the optimization procedure. A buckling optimization problem with different design cases is selected as a test problem and solved to evaluate the performance of the hybrid algorithm. The results are compared with the published data in the literature. Finally, a number of problems that include stacking sequence design cases for various inplane cyclic loadings are solved using the hybrid algorithm.

The results of the experimental correlation study indicate that the FTPF model can successfully predict the fatigue behavior of unidirectional and multidirectional composite laminates with different materials and configurations. Also, the results of the buckling optimization test problem show that the hybrid algorithm has superior or at least comparable performance in finding global optima compared to the best results in the literature. All these results demonstrate the applicability of the fatigue optimization procedure.

In fatigue optimization, multiple global optimum designs are obtained for most of the loading cases. It is seen that fatigue life is very sensitive to stress level in composite layers. The increase in the stress level considerably shortens the fatigue life of the laminates. Also, the loading combinations such as tension-tension, tension-compression, tension-tension-shear have a significant effect on fatigue life. This fact is especially seen in the tensioncompression loadings and the loadings combined with shear. The optimum stacking sequence designs are usually obtained for fatigue lives in the range of $10^{5}-10^{7}$ cycles. However, in high loading cases the algorithm yields optimum designs with fatigue life in the range of $10^{2}-10^{4}$ cycles. Besides, the comparison study between the optimization with conventional lamination using discrete fiber angles and the optimization with non-conventional lamination using integer fiber angles has shown that considerable fatigue life increase in stacking sequences are obtained by non-conventional lamination.

All these outcomes demonstrate the necessity and importance of design optimization practice of laminated composites for fatigue life advance. In this manner, the study shows in general that fatigue life, thus fatigue strength of laminated composites can seriously be improved by the appropriate fiber stacking sequence designs along with a reliable prediction method and an enhanced hybrid optimization algorithm.

\section{References}

[1] Vassilopoulos AP, Keller T. Fatigue of fiber-reinforced composites. SpringerVerlag; 2011.

[2] Adali S. Optimisation of fiber reinforced composite laminates subject to fatigue loading. Compos Struct 1985:3:43-55.

[3] Walker M. A method for optimally designing laminated plates subject to fatigue loads for minimum weight using a cumulative damage constraint. Compos Struct 2000;48:213-8.

[4] Ertas AH, Sonmez FO. Design optimization of fiber-reinforced laminates for maximum fatigue life. J Compos Mater 2014;48(20):2493-503.

[5] Muc A, Muc-Wierzgoń M. Discrete optimization of composite structures under fatigue constraints. Compos Struct 2015;133:834-9.

[6] Quaresimin M, Susmel L, Taljera R. Fatigue behaviour and life assessment of composite laminates under multiaxial loadings. Int J Fatigue 2010;32(1):2-16.

[7] Hashin Z, Rotem A. A fatigue failure criterion for fiber reinforced materials. J Compos Mater 1973;7:448-64.

[8] Rotem A. Fatigue failure of multidirectional laminate. AIAA J 1979;17 (3):271-7.

[9] Fawaz Z, Ellyin F. Fatigue failure model for fibre-reinforced materials under general loading conditions. J Compos Mater 1994;28(15):1432-51.
[10] Philippidis TP, Vassilopoulos AP. Fatigue strength prediction under multiaxial stress. J Compos Mater 1999;33(17):1578-99.

[11] Kawai M. A phenomenological model for off-axis fatigue behavior of unidirectional polymer matrix composites under different stress ratios. Compos Part A-Appl S 2004;35(7-8):955-63.

[12] Hashin Z. Failure criteria for unidirectional fiber composites. J Appl Mech 1980;47:329-34.

[13] Philippidis TP, Vassilopoulos AP. Complex stress state effect on fatigue life of GRP laminates. Part II, Theoretical formulation. Int J Fatigue 2002;24 (8):825-30.

[14] Tsai SW, Hahn HT. Introduction to composite materials. Lancaster: Technomic: 1980.

[15] Naderi M, Maligno AR. Fatigue life prediction of carbon/epoxy laminates by stochastic numerical simulation. Compos Struct 2012;94:1052-9.

[16] Lian W, Yao W. Fatigue life prediction of composite laminates by FEA simulation method. Int J Fatigue 2010;32:123-33.

[17] Jen MHR, Lee CH. Strength and life in thermoplastic composite laminates under static and fatigue loads. Part I: experimental. Int J Fatigue 1998;20 (9):605-15.

[18] Liu Y, Mahadevan S. Probabilistic fatigue life prediction of multidirectional composite laminates. Compos Struct 2005;69:11-9.

[19] Montgomery DC, Runger GC. Applied statistics and probability for engineers. John Wiley \& Sons Inc; 2003.

[20] Yogeswaran M, Ponnambalam SG, Tiwari MK. An efficient hybrid evolutionary heuristic using genetic algorithm and simulated annealing algorithm to solve machine loading problem in FMS. Int J Prod Res 2009;47(19):5421-48.

[21] Alarifi A, Liu Z, Erenay FS, Elkamel A, Croiset E. Dynamic optimization of lurgi type methanol reactor using hybrid GA-GPS algorithm: the optimal shell temperature trajectory and carbon dioxide utilization. Ind Eng Chem Res 2016;55:1164-73.

[22] Deveci HA, Aydin L, Artem HS. Buckling optimization of composite laminates using a hybrid algorithm under Puck failure criterion constraint. J Reinf Plast Comp 2016;35:1233-47.

[23] Holland JH. Adaptation in natural and artificial systems: an introductory analysis with applications to biology, control, and artificial intelligence. Oxford, England: U Michigan Press; 1975.

[24] Rao SS. Engineering optimization: theory and practice. John Wiley \& Sons Inc; 2009.

[25] Torczon V. On the convergence of pattern search algorithms. SIAM J Optim 1997; 7:1-25.

[26] Nicosia G, Stracquadanio G. Generalized pattern search algorithm for peptide structure prediction. Biophys J 2008;95(10):4988-99.

[27] Vassilopoulos AP, Georgopoulos EF, Keller T. Comparison of genetic programming with conventional methods for fatigue life modeling of FRP composite materials. Int J Fatigue 2008;30:1634-45.

[28] Al-Assaf Y, Kadi HE. Fatigue life prediction of unidirectional glass fiber/epoxy composite laminae using neural networks. Compos Struct 2001;53:65-71.

[29] Júnior RCSF, Neto ADD, Aquino EMF. Building of constant life diagrams of fatigue using artificial neural networks. Int J Fatigue 2005;27:746-51.

[30] Vassilopoulos AP, Georgopoulos EF, Dionysopoulos V. Artificial neural networks in spectrum fatigue life prediction of composite materials. Int J Fatigue 2007;29:20-9.

[31] Xiang KL, Xiang PY, Wu YP. Prediction of the fatigue life of natural rubber composites by artificial neural network approaches. Mater Des 2014;57:180-5.

[32] Salmalian K, Soleimani M, Rouhi S. Fatigue life modeling and prediction of GRP composites using multi-objective evolutionary optimized neural networks. MMMAS 2012;1(6):1-10.

[33] Riche RL, Haftka RT. Optimization of laminate stacking sequence for buckling load maximization by genetic algorithm. AIAA J 1993;31:951-6.

[34] Optimization ToolboxTM, MATLAB computer software in version R2016a, The Mathworks Inc; 2016.

[35] Soremekun G, Gürdal Z, Haftka RT, Watson LT. Composite laminate design optimization by genetic algorithm with generalized elitist selection. Comput Struct 2001:79:131-43.

[36] Rao ARM, Arvind N. A scatter search algorithm for stacking sequence optimisation of laminate composites. Compos Struct 2005;70:383-402.

[37] Rao ARM, Arvind N. Optimal stacking sequence design of laminate composite structures using Tabu embedded simulated annealing. Struct Eng Mech 2007;25:239-68.

[38] Rao ARM, Lakshmi K. Optimal design of stiffened laminate composite cylinder using a hybrid SFL algorithm. J Compos Mater 2012;46:3031-55.

[39] Rao ARM. Lay-up sequence design of laminate composite plates and a cylindrical skirt using ant colony optimization. Proc IMechE, Part G: J Aerospace Eng 2009:223:1-18.

[40] Rotem A, Nelson HG. Residual strength of composite laminates subjected to tensile-compressive fatigue loading. J Compos Technol Res 1990;12(2):76-84. 